\title{
Strategies for optimizing BioNano and Dovetail explored through a second reference quality assembly for the legume model, Medicago truncatula
}

Karen M. Moll ${ }^{1,2+}$, Peng Zhou ${ }^{3 \dagger}$, Thiruvarangan Ramaraj ${ }^{1}$, Diego Fajardo ${ }^{1}$, Nicholas P. Devitt', Michael J. Sadowsky ${ }^{4}$, Robert M. Stupar ${ }^{5}$, Peter Tiffin ${ }^{6}$, Jason R. Miller ${ }^{7}$, Nevin D. Young ${ }^{6}$, Kevin A. T. Silverstein $^{8}$ and Joann Mudge ${ }^{1^{*}}$

\begin{abstract}
Background: Third generation sequencing technologies, with sequencing reads in the tens- of kilo-bases, facilitate genome assembly by spanning ambiguous regions and improving continuity. This has been critical for plant genomes, which are difficult to assemble due to high repeat content, gene family expansions, segmental and tandem duplications, and polyploidy. Recently, high-throughput mapping and scaffolding strategies have further improved continuity. Together, these long-range technologies enable quality draft assemblies of complex genomes in a cost-effective and timely manner.

Results: Here, we present high quality genome assemblies of the model legume plant, Medicago truncatula (R108) using PacBio, Dovetail Chicago (hereafter, Dovetail) and BioNano technologies. To test these technologies for plant genome assembly, we generated five assemblies using all possible combinations and ordering of these three technologies in the R108 assembly. While the BioNano and Dovetail joins overlapped, they also showed complementary gains in continuity and join numbers. Both technologies spanned repetitive regions that PacBio alone was unable to bridge. Combining technologies, particularly Dovetail followed by BioNano, resulted in notable improvements compared to Dovetail or BioNano alone. A combination of PacBio, Dovetail, and BioNano was used to generate a high quality draft assembly of R108, a M. truncatula accession widely used in studies of functional genomics. As a test for the usefulness of the resulting genome sequence, the new R108 assembly was used to pinpoint breakpoints and characterize flanking sequence of a previously identified translocation between chromosomes 4 and 8, identifying more than $22.7 \mathrm{Mb}$ of novel sequence not present in the earlier A17 reference assembly.

Conclusions: Adding Dovetail followed by BioNano data yielded complementary improvements in continuity over the original PacBio assembly. This strategy proved efficient and cost-effective for developing a quality draft assembly compared to traditional reference assemblies.
\end{abstract}

Keywords: Genome assembly, Next generation sequencing, BioNano, Dovetail, PacBio, Medicago truncatula

* Correspondence: jm@ncgr.org

${ }^{\dagger}$ Equal contributors

${ }^{1}$ National Center for Genome Resources, 2935 Rodeo Park Drive East, Santa

Fe, NM 87505, USA

Full list of author information is available at the end of the article

(c) The Author(s). 2017 Open Access This article is distributed under the terms of the Creative Commons Attribution 4.0 International License (http://creativecommons.org/licenses/by/4.0/), which permits unrestricted use, distribution, and reproduction in any medium, provided you give appropriate credit to the original author(s) and the source, provide a link to the Creative Commons license, and indicate if changes were made. The Creative Commons Public Domain Dedication waiver (http://creativecommons.org/publicdomain/zero/1.0/) applies to the data made available in this article, unless otherwise stated. 


\section{Background}

Next generation sequencing technologies such as 454, Illumina, and SOLiD became available in the late 2000s $[1,2]\{$ Margulies, $2005 \# 113\}$. These technologies have the advantage of extremely high throughput and much lower cost per sequenced base compared to Sanger sequencing [3-8]. Long read sequencing technologies, such as PacBio and Oxford Nanopore, produce reads in the tens- of kilo-base range, much longer than what was possible even with traditional Sanger technology. However, they also have higher error rates, lower throughput, and higher costs per base compared to the short read technologies. Recently, PacBio throughput and cost per base have improved to the point that de novo plant genome assemblies using only PacBio are possible $[9,10]$.

Concomitantly, the throughput and cost of long-range scaffolding and mapping technologies that can increase continuity of an assembly have also improved dramatically. Traditional physical maps, dependent on expensive BAC library preparation, have given way to a variety of new technologies, including Opgen, Keygene, BioNano, and Nabsys maps [11-15]. BioNano is a high throughput optical mapping technology that utilizes endonucleases to nick long DNA molecules at the enzyme's recognition site, incorporating fluorescent nucleotides to obtain sequence-based patterns. The specific patterns are then used to assemble DNA molecules into a larger genome map, which can then be used to direct and improve a de novo genome assembly [16].

Genomic architecture analyses also can be achieved by sequencing libraries produced from chromatin proximity ligation methods such as Hi-C [17]. Dovetail Chicago libraries are similar to $\mathrm{Hi}-\mathrm{C}$ but rely on library preparation from in vitro rather than in vivo reconstituted chromatin that has been cross-linked and sheared. Dovetail Chicago libraries also use extraction of high molecular weight DNA extraction which limits input DNA length compared to $\mathrm{Hi}-\mathrm{C}$, which uses intact chromosomes. These libraries retain proximity signal with sequences physically close together being linked more often than those farther apart. This generates sequence pairs with insert sizes that can be as large as the size of the input DNA, typically $100 \mathrm{~kb}$, for use in scaffolding with Dovetail's in-house software [18].

Although BioNano and Dovetail are both long-range scaffolding technologies, there are several important differences. While both rely on restriction endonuclease digestions, different restriction enzymes are used for both technologies, potentially introducing different regional biases. Dovetail and BioNano also differ in the way they handle gaps. Dovetail does not attempt to size the gap, but instead adds $100 \mathrm{Ns}$ between scaffolds that it joins. By contrast, BioNano estimates gap size. Consequently, BioNano can appear to increase scaffold size more when the same scaffolds are joined with both technologies. In addition, BioNano does not automatically split sequences while Dovetail does. BioNano produces a file with possible chimeric sequences, but splitting of these sequences requires manual intervention by the user.

These new sequencing and mapping technologies have increased throughput, driven down costs, and introduced important technological advantages facilitating the sequencing of plant genomes, which are notoriously difficult due to large-scale duplications and repeats [19]. Indeed, these technologies are enabling the construction of multiple high quality plant genome assemblies $[4,6,7,9,20-29]$ and are now poised to increase the number of sequenced plant genomes even further.

Because legumes (family Fabaceae) are important in both agriculture and natural ecosystems, primarily due their capacity to form symbiotic relationships with nitrogen fixing bacteria, multiple genome assemblies are now available. Reference assemblies exist for lotus (Lotus japonicus) [30], soybean (Glycine max) [31], medicago (Medicago truncatula) [32], chickpea (Cicer arietinum) [33], mungbean (Vigna radiata) [34] and peanut (Arachis sp.) [10, 35]. Recently, multiple genome assemblies of a single plant species have begun to appear, enabling the identification of variation in genome content and structure segregating within species [36-40], including legumes [36, 39].

Medicago truncatula is a widely studied legume genome, especially in the area of plant-bacterial symbioses. Two Medicago accessions have been mainly used for genomic studies, R108 and A17 (Tadege et al. 2008, Young 2011). The relationship of R108 to A17, the accession used for generating the $M$. truncatula reference genome, makes it valuable both for a technology comparison and as a second M. truncatula assembly. Genotype $\mathrm{R} 108$ is one of the most distant $M$. truncatula accessions from A17 [41]. Relative to A17, R108 has much higher transformation efficiency, has a shorter generation time, and is easier to germinate, making it attractive for genetic studies [42]. Also, R108 is also important to the plant and symbiosis communities because it is the accession that was used to create a large Tnt1-insert population, widely used in functional analysis [42, 43]. Having two high quality references in Medicago therefore allowed us to perform comprehensive genome-scale comparisons between the two assemblies, revealing additional novel R108 sequences as well as increased fine-structure details of important rearrangement events compared to previous analyses using ALLPATHS-LG assemblies [39].

$M$. truncatula has a modest genome size, approximately $465 \mathrm{Mb}$ [44]. However, it also has an evolutionary history of whole genome duplications [45, 46] and 
frequent local duplications, which appear to be particularly common in this plant species [32], both of which make assembly difficult. We therefore generated and evaluated five combinations of PacBio, BioNano, and Dovetail technology to see how the technologies could complement each other and to explore differences in the ordering of technologies. Ultimately, we present a second, high quality reference genome for $M$. truncatula accession R108, based on an optimized combination of the three sequencing/mapping technologies.

\section{Results}

Assembly $\mathrm{Pb}$ was generated using $\sim 100 \mathrm{X}$ PacBio coverage and the FALCON assembler followed by Quiver polishing. Four additional assemblies were then created that had either BioNano (PbBn), Dovetail (PbDt), or both scaffolding technologies added onto the base assembly. The assemblies with both scaffolding technologies were created by applying BioNano and then Dovetail $(\mathrm{PbBnDt})$ or Dovetail and then BioNano (PbDtBn).

\section{Assembly continuity}

The $\mathrm{Pb}$ base assembly had just over 1000 contigs with no gaps in the sequence (Table 1). It totals just under $400 \mathrm{Mb}$ compared to $412 \mathrm{Mb}$ assembled in the $M$. truncatula A17 reference out of the estimated $465 \mathrm{Mb}$ genome size. The contig N50 for the $\mathrm{Pb}$ assembly is 3.77 $\mathrm{Mb}$ and the longest sequence is $13.59 \mathrm{Mb}$. We then added mapping or scaffolding technologies (BioNano and/or Dovetail) on top of this base assembly to improve scaffolding.

Both BioNano and Dovetail (PbBn or PbDt) technologies improved the PacBio only base assembly in similar ways (Table 1). The number of scaffolds decreased in both assemblies, dropping by 80 scaffolds in the $\mathrm{PbBn}$ assembly and 68 scaffolds in the PbDt assembly while having little effect on total scaffold length (Table 1). The PbBn assembly increased the scaffold length by approximately $1 \%$, adding 4.4 Mb, likely reflecting the fact that BioNano, unlike Dovetail, sizes the gaps it makes when joining sequences. Dovetail adds 100 Ns for each gap it creates, adding only $11.6 \mathrm{~kb}$ to the scaffold length.

The scaffold N50 s increased substantially for both the $\mathrm{PbBn}$ and $\mathrm{PbDt}$ assemblies, from $3.8 \mathrm{Mb}$ in the base $\mathrm{Pb}$ assembly to over $6.8 \mathrm{Mb}$ in both assemblies (Table 1). Although the scaffold N50 was slightly higher in the $\mathrm{PbDt}$ assembly (6.9 Mb vs $6.8 \mathrm{Mb}$ ), the N50 when adjusted for total genome size to allow for comparisons across assemblies (adjusted N50) dropped to $6.3 \mathrm{Mb}$ in the $\mathrm{PbDt}$ assembly but remained unchanged in the $\mathrm{PbBn}$ assembly. Maximum scaffold sizes increased in both assemblies, from $13.5 \mathrm{Mb}$ in the $\mathrm{Pb}$ assembly to $22.1 \mathrm{Mb}$ in the $\mathrm{PbBn}$ assembly and $19.3 \mathrm{Mb}$ in the PbDt assembly.

Adding a second technology to the $\mathrm{PbBn}$ and $\mathrm{PbDt}$ assemblies resulted in two assemblies that differed only in the order in which the BioNano and Dovetail technologies were applied. Overall, the PbBnDt and $\mathrm{PbDtBn}$ assemblies were very similar by scaffold size metrics (Table 1). Combining all three technologies resulted in slight decreases in the number of scaffolds, slight increases in total scaffold length, and large increases in scaffold N50 (Table 1). The increase in continuity was particularly striking, with the scaffold N50 nearly doubling to over $12 \mathrm{Mb}$ relative to the $\mathrm{PbBn}$ and $\mathrm{PbDt}$ assemblies and nearly tripling relative to the $\mathrm{Pb}$ base assembly. The maximum scaffold length was slightly larger in the PbBnDt assembly (30.4 Mb vs 27.3 Mb in the PbDtBn assembly), though the PbDtBn assembly had a slightly larger increase over its input assembly (PbDt).

As expected, given that neither BioNano nor Dovetail added a significant amount of sequence data, the number of contigs, contig lengths, and N50 s, were nearly identical for all five assemblies (Table 1). The only substantial change to the contig stats was a slight increase in the number of contigs when Dovetail technology was used, due to the breaking of chimeric contigs (Table 1).

Table 1 Number and characteristics of contigs and scaffolds for each of the five assemblies

\begin{tabular}{|c|c|c|c|c|c|}
\hline & PacBio (Pb) & $\begin{array}{l}\text { PacBio BioNano } \\
(\mathrm{PbBn})\end{array}$ & $\begin{array}{l}\text { PacBio Dovetail } \\
\text { (PbDt) }\end{array}$ & $\begin{array}{l}\text { PacBio BioNano } \\
\text { Dovetail (PbBnDt) }\end{array}$ & $\begin{array}{l}\text { PacBio Dovetail } \\
\text { BioNano (PbDtBn) }\end{array}$ \\
\hline Assembly software & FALCON & FALCON Irys & FALCON HiRise & FALCON Irys HiRise & FALCON HiRise Irys \\
\hline Contigs & 1,073 & 1,073 & 1,121 & 1,125 & 1,121 \\
\hline Contig Length & $396,973,838$ & $396,973,942$ & $396,973,838$ & $396,973,942$ & $396,973,934$ \\
\hline Contig $\mathrm{N} \mathrm{O}^{\mathrm{a}}$ & $3,768,504$ & $3,768,512$ & $3,768,504$ & $3,768,512$ & $3,768,504$ \\
\hline Scaffolds & 1,073 & 993 & 1,005 & 965 & 942 \\
\hline Scaffold Length & $396,973,838$ & $401,421,527$ & $396,985,438$ & $401,429,527$ & $399,955,467$ \\
\hline Maximum Scaffold Length & $13,488,151$ & $22,885,216$ & $19,275,758$ & $12,137,306$ & $12,557,854$ \\
\hline Scaffold N50 ${ }^{a}$ & $3,768,504$ & $6,819,834$ & $6,895,511$ & $12,137,306$ & $12,557,854$ \\
\hline
\end{tabular}

${ }^{a} \mathrm{~N} 50 \mathrm{~s}$ were also adjusted to use an assembly length of $400 \mathrm{Mb}$ for all assemblies in order to facilitate comparisons across assemblies. Scaffold and contig N50 s adjusted for a $400 \mathrm{Mb}$ assembly size were identical to unadjusted N50 s shown above, except for the PbDt scaffold N50 for which the adjusted N50 was 6,348,449 nt 


\section{Assembly completeness}

To assess assembly completeness we examined the number of genomic reads that were captured by the assembly. We used PacBio reads, which were used to create the assemblies, as well as Illumina reads, which represent an independent read set, that were captured by the assemblies. The base $(\mathrm{Pb})$ assembly captured $91.8 \%$ of the PacBio reads and $96.8 \%$ of the Illumina reads. Moreover, $95.7 \%$ of the Illumina reads aligned as pairs with expected orientation and distance, indicating that, at least on the local scale, the assembly is accurate.

Because BioNano and Dovetail are scaffolding technologies, they are not expected to add a substantial amount of additional sequence, but rather to organize the assembly sequences into longer scaffolds. Indeed, the estimates of assembly completeness obtained through read capture did not change meaningfully upon the addition of these technologies (Additional file 1: Table S1).

\section{Gene space completeness}

In order to investigate the completeness of the gene space in the five assemblies we determined rates of capture for conserved single-copy eukaryotic genes (BUSCO) [47] and an R108 transcriptome assembly, and assessed MAKER-P annotations. Because completeness results for all 5 assemblies were quite similar we discuss only results for the $\mathrm{Pb}$ base assembly and present results for the other assemblies in the supplement (Additional file 1: Table S2). The BUSCO analysis indicates that the base assembly $(\mathrm{Pb})$ captured nearly all of the genes $(878$ of the 956 genes in the dataset; $91.8 \%$ ). Nearly 16\% (151) of the putative single-copy genes in the BUSCO database were duplicated within the assemblies. These putative duplicates might be due to true duplications in the R108 genome or they might be due to artificial redundancy in the assembly. Even though the BUSCO gene groups are generally single copy, given plant genome duplication rates it isn't surprising that some of the genes are duplicated.

In addition to looking at capture of conserved genes, we also looked at capture of an R108 RNA-Seq assembly that was produced independently of the genome. Assembly completeness results were similar to those seen with BUSCO, with approximately 92\% $(94,519)$ transcripts captured. However, as would be expected, the duplication rate was much higher than that seen in BUSCO, which specifically focuses on single copy genes. In the R108 transcript assembly, 37,929 transcripts (37\% of total, $40.1 \%$ of aligned transcripts) were duplicated.

Finally, we analyzed the total number of genes predicted from MAKER-P. There were 54,111 genes compared to 50,894 gene loci in Mt4.0 (accession A17). This gives additional confirmation that the gene space is largely complete. Further, there may be additional genes in the R108 Pb assembly not found in A17 (see below).

\section{Joins and breaks}

When characterizing the joins made by BioNano and Dovetail, some interesting trends emerged (Additional file 1: Table S3). Dovetail joined more scaffolds when applied to the base $(\mathrm{Pb})$ assembly compared to BioNano. Dovetail joined $172 \mathrm{~Pb}$ scaffolds into 64 PbDt scaffolds while BioNano joined $140 \mathrm{~Pb}$ scaffolds into $50 \mathrm{PbBn}$ scaffolds. The same trend of more joins for Dovetail compared to BioNano held when adding a second scaffolding or mapping technology. Dovetail joined 114 $\mathrm{PbBn}$ scaffolds into $45 \mathrm{PbBnDt}$ scaffolds and BioNano joined $96 \mathrm{PbDt}$ scaffolds into $33 \mathrm{PbDtBn}$ scaffolds. For the two contrasting assemblies created with all technologies, the two rounds of scaffolding resulted in a total of 254 scaffolds joined in the PbBnDt assembly and 268 scaffolds joined in the PbDtBn assembly, a difference of just over $5 \%$. While Dovetail joined more scaffolds, BioNano had a higher average number of scaffolds per join (Additional file 1: Table S3).

To determine the characteristics of scaffolds that were being joined, we pulled out scaffolds from the input assembly that were joined by either technology in either round (Table 2, Additional file 1: Table S4). The biggest difference between the two technologies was in the ability to join shorter scaffolds. Dovetail was able to join scaffolds as short as 4765 nucleotides into a larger super-scaffold (in both rounds 1 and 2), whereas the minimum scaffold size that BioNano was able to join was 172,295 in round 1 and 98,093 in round 2. To further understand the ability of Dovetail to join smaller contigs, we quantified the number of input scaffolds less than $100 \mathrm{~kb}$ that each technology was able to join

Table $\mathbf{2}$ Characteristics of input scaffolds that were joined by BioNano and/or Dovetail

\begin{tabular}{lllll}
\hline Assembly & $\mathrm{Pb}->\mathrm{PbDt}$ & $\mathrm{Pb}->\mathrm{PbBn}$ & $\mathrm{PbDt}->\mathrm{PbDtBn}$ & $\mathrm{PbBn}->\mathrm{PbBnDt}$ \\
\hline Scaffolds & 172 & 140 & 96 & 114 \\
Max Scaffold & $13,488,151$ & $13,488,151$ & $19,275,758$ & $22,885,216$ \\
Scaffold N50 & $3,957,684$ & $3,698,567$ & $6,895,511$ & $6,819,834$ \\
Scaffold N90 & 854,372 & 929,179 & $1,425,957$ & $1,427,073$ \\
Min Scaffold & 4,765 & 172,295 & 98,093 & 4,765 \\
Total Scaffold Length & $307,402,024$ & $293,002,927$ & $260,974,793$ & $289,680,947$ \\
\hline
\end{tabular}


(Additional file 1: Table S4). Dovetail joined 35 sub$100 \mathrm{~kb}$ scaffolds (17 in round 1 and 18 in round 2). BioNano, on the other hand joined only 1 sub-100 kb scaffold total (in round 2), and that scaffold was nearly $100 \mathrm{~kb}(98,093 \mathrm{nt})$. Clearly, Dovetail is better at incorporating short scaffolds less than $100 \mathrm{~kb}$.

While Dovetail appears to be better at incorporating shorter scaffolds, it also appears to more effectively join longer scaffolds. When only scaffolds $>=100 \mathrm{~kb}$ cutoff were examined, Dovetail joined 253 input scaffolds and BioNano joined 237 across both rounds. Similarly, when only very large scaffolds were examined $(>=1 \mathrm{Mb})$ Dovetail joined 141 input scaffolds and BioNano joined 128 across both rounds. Dovetail had a higher number of joins at each cutoff when the data were broken down by each round as well (data not shown).

To identify similarities between the two technologies, we determined whether some of the joins made were the same between BioNano and Dovetail. We focused on the first round, where each technology was added onto the $\mathrm{Pb}$ assembly, looking for cases where the same $\mathrm{Pb}$ scaffolds were joined into a super-scaffold. There were 47 $\mathrm{Pb}$ input scaffolds that were scaffolded by both BioNano and Dovetail, resulting in 21 scaffolds in the PbDt assembly and 20 scaffolds in the $\mathrm{PbBn}$ assembly. The fact that these joins were made by two independent technologies improves our confidence in these joins. Given that there were also joins made that were unique to both technologies supports the increased continuity and additional joins that we are seeing in assemblies that have both technologies added.

In order to determine whether Dovetail was breaking apart scaffolds that BioNano had previously created by merging $\mathrm{Pb}$ scaffolds, we looked further into the Dovetail breaks. In other words, we asked whether any of the joins made by BioNano when generating the $\mathrm{PbBn}$ assembly were subsequently split by Dovetail when applied to the $\mathrm{PbBn}$ assembly to generate the $\mathrm{PbBnDt}$ assembly. From the merged scaffolds generated in the $\mathrm{PbBn}$ assembly, only $8 \mathrm{PbBn}$ scaffolds were broken by Dovetail in the PbBnDt assembly and no breaks occurred directly inside the gaps that had been generated by BioNano (median distance from gap was 137,686 nt). We generally found read support spanning these regions, with half or more of the alignments having equally good hits to other regions of the assembly (data not shown). This indicates that these were large repetitive regions and it was difficult to say confidently whether the region should be joined (BioNano correct) or broken (Dovetail correct).

\section{Joins and breaks in relation to $\mathrm{A} 17$}

We used alignments of first round assembly scaffolds $(\mathrm{PbBn}$ and $\mathrm{PbDt})$ to $\mathrm{A} 17$ to predict whether scaffold joins were correct. If joined pieces of a scaffolds mapped to the same A17 chromosome, this lends support for the join. Because of the evolutionary distance between R108 and $\mathrm{A} 17$, rearrangements are expected, so a negative result doesn't necessarily mean the join is incorrect. However, vastly different rates of A17 synteny between scaffold joins made by BioNano and Dovetail would suggest better accuracy for one of the technologies.

Scaffolds joined by BioNano mapped to the same A17 chromosome at a rate of $78.57 \%$ while those joined by Dovetail mapped to the same A17 chromosome at a rate of $93.75 \%$. This suggests that Dovetail had a better accuracy than BioNano. Scaffolds with joins that were supported by both BioNano and Dovetail appear to be of higher accuracy based on alignments to A17. For BioNano, while over half of joins (54.54\%) were from scaffolds that had similar joins by Dovetail, only $20.00 \%$ of joins that mapped to different A17 chromosomes were supported by a similar Dovetail scaffold. This resulted in a $90.91 \%$ of Dovetail-supported BioNano joins that mapped to the same A17 chromosome, an increase of $12.34 \%$ over all BioNano joins. Dovetail, had more joins than BioNano (see above), with $36.67 \%$ of the joins supported by a similar BioNano scaffold. A similar percentage was seen in the number of BioNano-supported Dovetail joins compared to all Dovetail joins (33.33\%), resulting in 94.29\% of BioNano-supported Dovetail joins aligning to a single A17 chromosome, representing an increase of $0.54 \%$.

Finally, we looked at A17 synteny in the eight $\mathrm{PbBn}$ scaffolds that were subsequently broken by Dovetail in the $\mathrm{PbBnDt}$ assembly. Three of the scaffolds had input pieces that mapped to chromosome U (unknown), making it difficult to determine A17 synteny and indicating that repetitive sequence is likely that made it difficult to make a chromosome assignment. Of the other 5 scaffolds, 3 mapped to the same A17 chromosome, supporting the BioNano join and 2 mapped to different chromosomes, supporting the subsequent Dovetail break.

\section{Gaps}

The sizing of gaps in BioNano versus the addition of 100 nts in Dovetail, resulted in an increase in the amount of nucleotides added to the total scaffold length in the first round for BioNano compared to Dovetail (Table 1).

In order to see how the gap strategies of BioNano and Dovetail interact, we analyzed the second round assemblies ( $\mathrm{PbBnDt}$ and $\mathrm{PbDtBn}$ ), which have both technologies incorporated but with differing order. When a second scaffolding or mapping technology was added to an assembly that already incorporated the other technology, the gaps from the first technology were carried over intact. As noted above, Dovetail sometimes broke apart scaffolds that BioNano had put together. However, when breaking these scaffolds, Dovetail never broke the 
scaffolds within the gap generated by BioNano but rather broke it in a nearby position. In assemblies where BioNano was added to the PbDt assembly, the minimum gap size that BioNano introduced was $500 \mathrm{nt}$. This minimum size might be because $500 \mathrm{nt}$ is the minimum gap BioNano can span. Alternatively, given that the assemblies are all based upon PacBio data, it may be that smaller gaps were easily bridged by the PacBio data itself.

The assemblies with both BioNano and Dovetail ( $\mathrm{PbBnDt}$ and $\mathrm{PbDtBn}$ ) ended up with a similar number of captured gaps (Table 3). The maximum gap length was over $647 \mathrm{~kb}$, generated when adding BioNano onto the $\mathrm{Pb}$ assembly. Although Dovetail doesn't size its gaps, given the insert size of $\sim 100 \mathrm{~kb}$, it is likely that most of the gaps fall below this range. BioNano, with a gap N50 of 171,515 (Table 3), therefore was able to jump across larger distances than Dovetail.

A similarly sized gap generated when adding BioNano onto the $\mathrm{PbDt}$ assembly traces back to the same $\mathrm{Pb}$ scaffolds as the join made by BioNano on the $\mathrm{Pb}$ assembly. Finally, the total gap length varies. Among those assemblies that contain sized gaps (PbBn, $\mathrm{PbBnDt}$, and $\mathrm{PbDtBn}$ ), the $\mathrm{PbDtBn}$ assembly has considerably fewer nts in gaps compared to the other two. This is somewhat surprising given the fact that this assembly has the most gaps of any assembly and that there were more joins made over the two rounds in the PbDtBn assembly (268) than over both rounds in the PbBnDt assembly (254) (Additional file 1: Table S3). Overall, the gap sizes in PbDtBn are smaller (Table 3), accounting for the lower number of nts in gaps.

Finally, in order to surmise the nature of sequence in the gaps and why contigs stop instead of continuing on, we looked at the sequence flanking the gaps $(10 \mathrm{~kb})$. Interestingly, the joins made by BioNano and Dovetail (and the breaks made by Dovetail) were enriched for repetitive sequence in the regions flanking the gap introduced with the join (Additional file 1: Figure S1). BioNano and Dovetail both appear to be able to jump across larger repetitive regions than is possible with PacBio reads. In other words, the value of the two

Table 3 Characteristics of the gaps introduced into the assemblies by BioNano and Dovetail. Note, there are no gaps in the Pb only base assembly so it is not included

\begin{tabular}{lllll}
\hline & $\mathrm{PbBn}$ & $\mathrm{PbDt}$ & $\mathrm{PbBnDt}$ & $\mathrm{PbDtBn}$ \\
\hline Captured Gaps & 80 & 116 & 160 & 179 \\
Max Gap & 647,836 & 100 & 647,836 & 647,022 \\
Min Gap & 500 & 100 & 100 & 100 \\
Mean Gap & 55,595 & 100 & 27,847 & 16,657 \\
Gap N50 & 171,515 & 100 & 171,515 & 105,896 \\
Total Gap Length & $4,447,585$ & 11,600 & $4,455,585$ & $2,981,533$ \\
\hline
\end{tabular}

technologies is often in their ability to bridge across repetitive regions that PacBio reads cannot currently cross.

\section{Ordering of technologies}

The ordering of the scaffolding or mapping technologies made a difference to the continuity and completeness statistics (Table 1, Additional file 1: Tables S1 and S2). Using Dovetail before BioNano provides multiple benefits. The fact that Dovetail breaks chimeric scaffolds automatically means that using it up front provides a cleaner assembly template for BioNano. Dovetail's ability to scaffold much smaller pieces of DNA compared to BioNano means that if Dovetail is used up front, more joins will be made and a better base sequence assembly constructed.

\section{Final assembly draft}

In order to create the best reference assembly, we gapfilled the PbDtBn assembly using PBJelly (named R108 version 1.0, Table 4). The PbDtBn assembly was chosen because it had slightly better assembly stats compared to PbBnDt (Table 1, Additional file 1: Tables S1 and S2). For the five preliminary assemblies interrogated above, we did not do any gap filling or polishing (except that the base assembly was polished with Quiver) because these methods would obscure the effects that the BioNano and Dovetail technologies were having on the assembly process. Nevertheless, PBJelly was used for gap-filling as well as super-scaffolding on the final assembly draft in order to improve continuity. While gap filling can be over-aggressive especially if flanking sequences are repetitive, having some sequence, even if not perfect, is often better than having just Ns. In addition, using Dovetail and then BioNano enabled us to use independent data to bring scaffolds together and size the gap between them, making us more confident with doing gap-filling.

PBJelly was able to fill many of the captured gaps, increasing the continuity of the PbDtBn assembly (Tables 1 and 4). In total, it filled in 415 of 522 gaps (79.50\%). As expected, gap-filling was able to fill far more small than large gaps, resulting in an increase of the gap N50 from 12,335 nt to 110,194 nt, a nearly 9fold increase. The latter is much longer than typical

Table 4 Assembly Statistics for R108 version 1.0 (PbDtBn PBJelly gap filled) and its input assembly (PbDtBn)

\begin{tabular}{lll}
\hline & R108 $\vee 1.0$ & PbDtBn \\
\hline Contigs & 1,016 & 1,121 \\
Contig Length & $399,348,944$ & $396,973,934$ \\
Contig N50 & $5,925,378$ & $3,768,504$ \\
Scaffolds & 909 & 942 \\
Scaffold Length & $402,065,285$ & $399,955,467$ \\
Scaffold N50 & $12,848,239$ & $12,557,854$ \\
\hline
\end{tabular}


PacBio reads and may represent repeats that were too long to span with these reads. The total gap length was only reduced by $8.82 \%$ despite the fact that $79.50 \%$ of the gaps were filled, again reflecting the preferential filling of small gaps. Nevertheless, continuity is much improved. The number of contigs dropped by $\sim 12 \%$ to just over 1000 (1016 contigs), and the contig N50 increased from $3,768,504 \mathrm{nt}$ to $5,925,378 \mathrm{nt}$, representing an increase of $57.23 \%$. Gap filling had little effect on the number of scaffolds, scaffold N50, or total assembly size (differences between gap filled and ungapped assemblies were $<0.5 \%$.

The completeness stats of the gap filled assembly improved slightly relative to the $\mathrm{PbDtBn}$ assembly before gap-filling (Additional file 1: Tables S1 and S2). The final draft R108 v 1.0, assembly captured $93.2 \%$ of $\mathrm{Pb}$ reads and $96.8 \%$ of Illumina reads. Of the original Illumina readset, $95.8 \%$ were not only mapped but also properly paired, indicating that the assembly has captured most of the genome. The R108 v 1.p assembly has captured most of the gene space, with estimates ranging from $92.3 \%$ for the transcript assembly to $95.2 \%$ for the BUSCO assembly, and 55,706 genes predicted MAKERP. Overall, this final draft of the R108 assembly captures nearly all the assembly and gene space.

\section{Novel sequences revealed by the R108 assembly}

A new high quality reference sequence for R108 allowed a side-by-side comparison of two Medicago accessions (A17 and R108). We were able to build chromosome-level synteny blocks between R108 and A17. We also found extensive novel sequence in the R108 assembly that was not part of the A17 reference assembly (Table 5). There was nearly $23 \mathrm{Mb}$ of R108 assembly sequence that could not be found in the A17 assembly. This represents $5.7 \%$ of the nucleotides in the R108 genome. These "novel" sequences are likely a mix of sequences that are truly novel in the R108 genome as well as sequences that are present in both genomes but have diverged beyond our ability to detect them or sequences that are in the A17 genome but didn't make it into the A17 assembly. Out the nearly $23 \mathrm{Mb}$ of novel R108 sequence, 1.6 Mb represent novel R108 coding

Table 5 R108 v 1.0 assembly characteristics in comparison to the $\mathrm{A} 17$ reference assembly

\begin{tabular}{lll}
\hline & Nucleotides & \% Nucleotides \\
\hline Total Bases & $399,348,955$ & $100.00 \%$ \\
Repetitive & $96,760,262$ & $24.23 \%$ \\
Alignable to A17 & $366,489,898$ & $91.77 \%$ \\
Bases in Synteny with A17 & $283,853,354$ & $71.08 \%$ \\
Novel Sequences vs A17 & $22,763,508$ & $5.70 \%$ \\
Novel Coding Sequences vs A17 & $1,623,097$ & $0.41 \%$ \\
\hline
\end{tabular}

sequence that could not be found in the A17 assembly, values quite similar to those observed with an earlier ALLPATHS-LG [48] assembly of R108 [39]. These regions contain candidate R108-specific genes or gene that were deleted from A17 or arose independently in the R108 lineage.

\section{Chromosomal-scale translocation}

Although R108 is phylogenetically distant from A17 compared to other accessions, we were able to align more than $280 \mathrm{Mb}$ of syntenic regions in both genomes (Table 5), representing over 70\% of the R108 assembly. These numbers also correspond well with sequence comparisons based on an earlier ALLPATHS-LG assembly of R108 [39]. Within these synteny blocks, extensive variations were discovered including single nucleotide changes, small insertions and deletions, as well as large structural changes such as inversion and translocation. While most structural changes were TE-related and only involve small local regions, we identified two large rearrangements on chromosomes 4 and 8 between R108 and A17. Through synteny comparison, we found one R108 scaffold (scf005, 16.4 Mb) spanning the upper arm of chromosome 4 and the lower arm of chromosome 8 in A17, and another two scaffolds (scf015, 12.0 Mb and scf002, 17.6 Mb) together spanning the upper arm of chromosome 8 plus the lower arm of chromosome 4 (Fig. 1), indicating a chromosomal-scale translocation between the reference Medicago accession (A17) and the widely-used R108 accession.

Previously, Kamphuis et al. reported a rearrangement between linkage groups 4 and 8 in the reference accession A17 relative to other accessions [49]. Using genetic markers and linkage mapping, the authors hypothesized a chromosomal-scale translocation private to A17 which involves the lower arms of chromosomes 4 and 8 [49]. To date, however, the physical location of the rearrangement has not been determined and, in fact, the rearrangement itself has not been elaborated through genome sequencing. Lack of high quality genome assemblies of non-A17 accessions certainly hindered such whole genome comparison. However, even with the whole genome assemblies available (including the earlier R108 ALLPATHS-LG assembly), it is still difficult to fully resolve rearrangement events at such chromosomal scale given the relatively short scaffold span of most sequencing and assembly techniques. Figure 2 clearly illustrates the improvements in resolving large-scale structural variation using long PacBio reads together with scaffolding or mapping technologies such as Dovetail and BioNano, over traditional Illumina-based assembly or assembly based on PacBio reads alone. Using the same synteny pipeline we aligned the Illumina-based R108 assembly, assembled with ALLPATHS-LG [48], to 


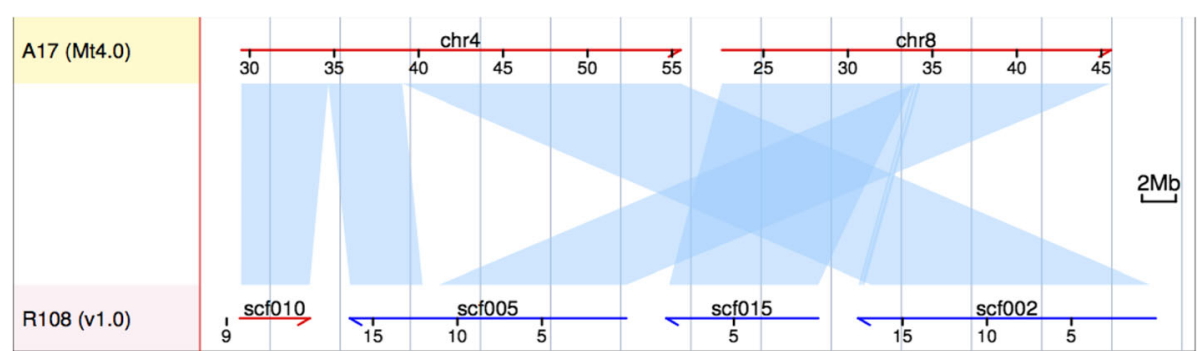

Fig. 1 Synteny alignment of partial chromosomes 4 and 8 between A17 and R108 confirms rearrangement of the long arms of the chromosomes

A17. The rearrangement region $(\sim 50 \mathrm{Mb})$ on chromosomes 4 and 8 was split into $\sim 30$ independent scaffolds in the ALLPATHS-LG R108 assembly (Fig. 2, top panel). The PacBio-based assembly $(\mathrm{Pb})$, on the other hand, captured the region in $\sim 10$ scaffolds and partially resolved the breakpoint on chromosome 4 (Fig. 2, middle panel). With the aid of BioNano and Dovetail technologies, the affected region was captured in four long scaffolds in the final R108 assembly (PacBio + Dovetail + BioNano) with all breakpoints clearly resolved (Fig. 2, bottom panel). We were able to pinpoint exact breakpoints of the translocation to a single region on chromosome 4 and three regions on chromosome 8 , something that could not be done with the Illumina-based ALLPATHS-LG assembly (Fig. 3). Interestingly, each of the four breakpoints involves a gap (i.e., 'N's) in the A17 reference, with one $7.5 \mathrm{kbp}$ gap and three $100 \mathrm{bp}$ gaps, the latter representing gaps of undetermined size (Haibao Tang, personal communication). These gaps indicate that the regions in and around the rearrangement breakpoints are structurally unstable, repetitive and/or difficult to assemble even using a BAC-by$\mathrm{BAC}$ approach. We found numerous transposable element genes near the breakpoints, including a reverse transcriptase, a GAG-pre integrase and a cluster of 6 transferases

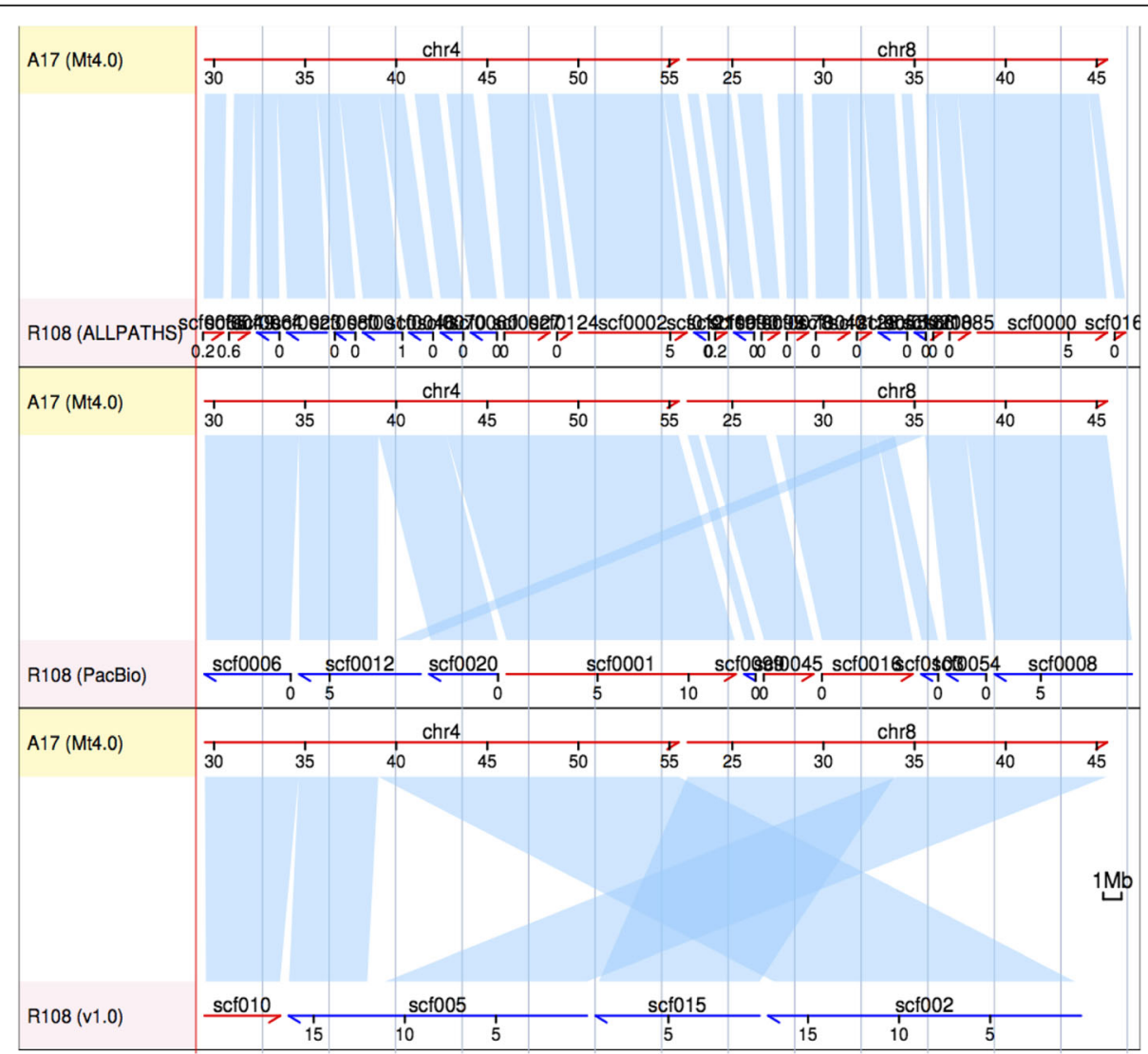

Fig. 2 Synteny alignment of partial A17 chromosomes 4 and 8 against syntenic regions in the R108 Illumina-based assembly (top panel), PacBio-based assembly ( $\mathrm{Pb}$, middle panel) as well as the gap-filled PbDtBn (v1.0) assembly (bottom panel) 


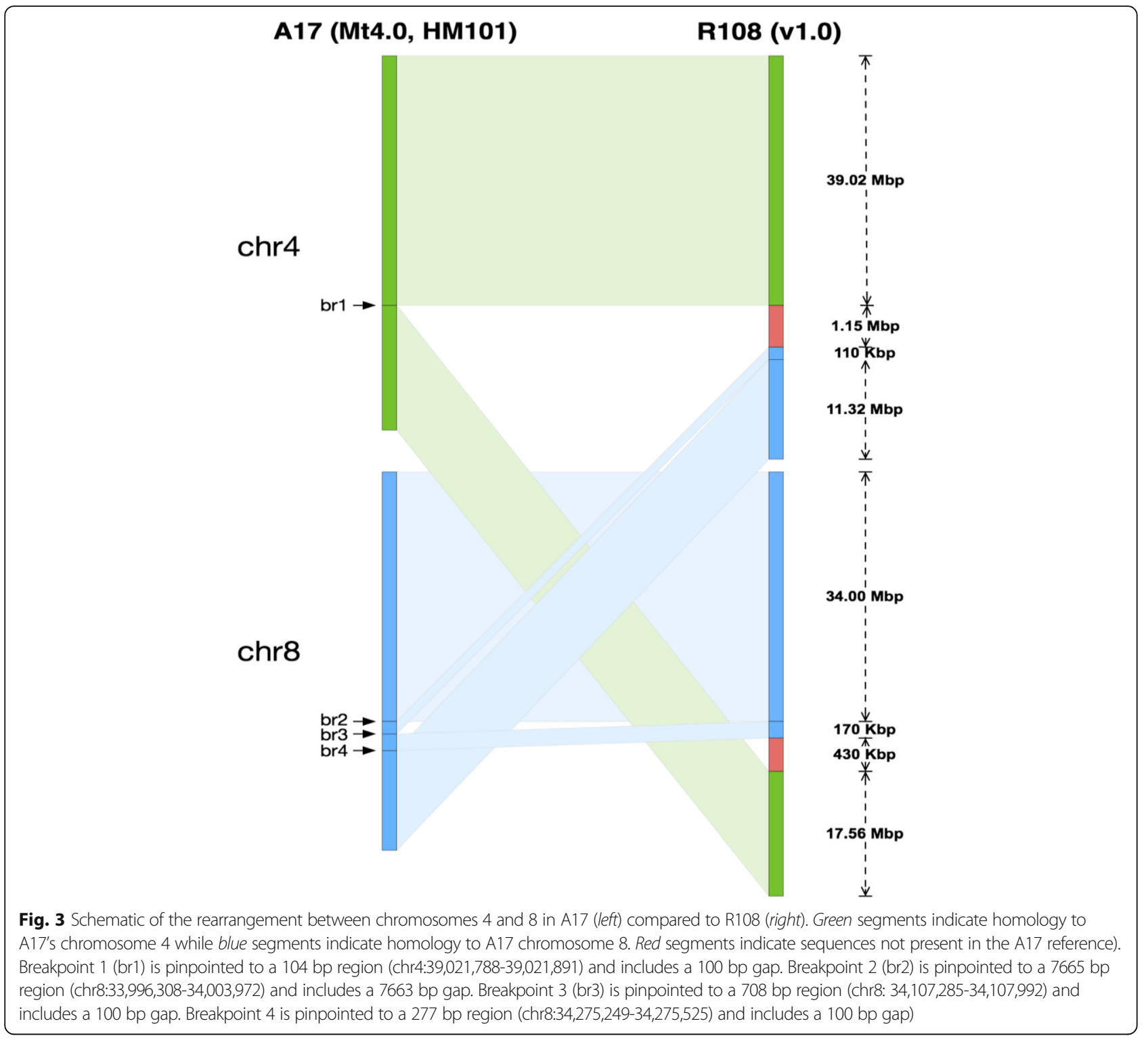

near breakpoint 1, two helicases around breakpoint 2, two retrotransposons $(\mathrm{UBN} 2)$ and two reverse transcriptases around breakpoint 3, and a MULE transposase right next to breakpoint 4. Intriguingly, a cluster of at least $10 \mathrm{CC}$ NBS-LRRs was found both upstream and downstream of breakpoint 2, and two CC-NBS-LRRs were also found right next to breakpoint 3 , possibly suggesting a structural role of these resistance genes in plant genomes.

In addition to the translocation, we noticed two large stretches of R108 sequences $(1.15 \mathrm{Mb}$ and $430 \mathrm{~Kb})$ downstream from the translocation breakpoints on chromosome 4 and 8 (Fig. 3 red segments) that didn't have a syntenic match in A17. The chromosome 4 insertion in $\mathrm{R} 108$ is $\mathrm{a} \sim 1 \mathrm{Mb}$ region with no synteny to A17 and right next to the chr4-8 translocation breakpoint. Both the translocation and insertion are found in several other accessions including HM034 and HM185 using a similar synteny comparison approach (data not shown). It is thus likely that the translocation is private to A17, which is consistent with [49], and this large insertion in R108 actually represents a private deletion in A17 which is expected to be found in the majority of $M$. truncatula accessions.

Further examination revealed that most of the insertion is novel. A total of $623 \mathrm{kbp}$ of novel segments that do not align anywhere in A17 were identified in this region with 136 genes found in this region (Additional file 1: Table S5).

\section{Discussion}

This work represents the first published example we are aware of examining multiple next generation scaffolding and mapping technologies in all possible combinations 
with a comparative analysis of their contributions. PacBio long reads combined with BioNano and Dovetail technologies have allowed us to generate a second, reference quality assembly for the model legume, $M$. truncatula, in the functionally-important R108 accession. In the process, we discovered important insights into how these technologies overlap and complement each other enabling us to propose an optimal strategy for their incorporation.

\section{Novel sequence was found in the R108 assembly}

Long reads improve the continuity of assemblies $[20,50-54]$. However, continuity is only one advantage of using long reads. The long reads help to correctly capture ambiguous regions of the genome in the assembly, including repeats and tandemly duplicated genes. Locally duplicated genes can be especially problematic as they are often collapsed or over-expanded in Illumina-only or even Illumina/PacBio hybrid assemblies (Miller et al., submitted). Using PacBio long reads, therefore, results in capture of additional sequence that is not possible with short reads. In addition, we capture accession specific sequences as well. In total, over $22 \mathrm{Mb}$ of novel sequence, including 1.6 Mb of coding sequence were identified.

\section{Technologies made similar continuity gains and are valuable individually}

Similar continuity gains were made by each technology in each round, as was seen in [6]. Both technologies improved the base $\mathrm{Pb}$ assembly, improving the $3.8 \mathrm{Mb}$ scaffold N50 of the Pb assembly to just over $6.8 \mathrm{Mb}$ (Table 1). Indeed, many of the same joins were made between both of the technologies. Both technologies, individually, were valuable in increasing continuity.

Despite the challenges of assembly the $M$. truncatula genome, with its history of whole genome duplication and high rate of locate duplication, there are many plant genomes that are much more complicated than the $500 \mathrm{Mb}$, largely homozygous Medicago truncatula genome. Increases in genome size, repetitive content, and the number of tandem, segmental, or whole genome duplications will change the dynamics of the assembly and the contributions of the technologies. In Medicago described here, the PacBio assembly came together quite well, making the improvements when using BioNano and Dovetail less dramatic than they might have been. As genome complexity increases, including repeat and duplication content, coherent PacBio assemblies become increasingly difficult. As PacBio assemblies become more fragmented with increased genome complexity, we expect that the improvement in the assembly when adding BioNano and/or Dovetail will become increasingly crucial, leading to greater relative improvements, even while becoming more challenging. The assembly improvement with both technologies should follow similar patterns with increased genome complexity until extremely high levels of complexity, especially repeat size, become limiting even for these technologies.

\section{Further gains were made using both technologies}

Though similar gains were seen when using either scaffolding or mapping technology, the use of both technologies together increased continuity gains and join numbers further (Table 1 and Additional file 1: Table S3) [6]. With a combined approach the two technologies were complementary by enabling additional joins than either Dovetail or BioNano could make independently. Using both scaffolding technologies in either order (PbDtBn or PbBnDt) increased the scaffold N50 to just over $12.1 \mathrm{Mb}$ (Table 1).

One explanation for the complementarity between the two technologies may be a function of the differences in biases of the two technologies. BioNano's information content is in restriction sites and the distances between them. As such, BioNano is highly dependent on the motif density of the restriction enzymes used [55, 56], which can vary within a genome. Genomic regions where motif density is high become "fragile sites", that destabilize the DNA and resulting in limited or no coverage in the maps, and breaks in the genome map contigs $[5,8,16,56]$. In these regions scaffolding of the assembly simply cannot occur. By contrast, regions of the genome with too low of a density of cutting sites also will result in low label density and missed join opportunities (a minimum of eight restriction sites is required in each DNA molecule, which is a minimum of $150 \mathrm{~kb}$ ).

Dovetail is based on Hi-C technology, an extension of chromosome conformation capture, which has its own documented biases $[57,58]$. Dovetail's information content is "contact probabilities," indicating the probability that any two regions in the genome will be brought together during the ligation stage and is inversely correlated with distance. Dovetail, which incorporates Illumina sequencing, also inherits biases in next generation sequencing and alignment, such as biases in the amplification, shearing and mapping steps.

\section{Join accuracy appears to be higher in dovetail compared to BioNano}

Using A17 synteny as a proxy for accuracy of joined R108 scaffolds, Dovetail had a much higher percentage of joins mapping to the same A17 chromosome compared to BioNano (93.75 vs $78.57 \%$ ), suggesting that accuracy is higher in Dovetail than in BioNano. Further, when looking at joins in scaffolds supported by both technologies, Dovetail-supported BioNano joins mapped to the same A17 chromosome 90.91\%, an increase of $12.34 \%$ over all BioNano joins. This suggests that 
Dovetail confirmation increases the accuracy of BioNano joins. BioNano-supported Dovetail joins, however, increased mapping to the same A17 chromosome by only 0.54\%, suggesting that BioNano confirmation did little to improve Dovetail accuracy.

These data argue that Dovetail joins are more accurate than BioNano joins. However, we cannot rule out that the possibility that the larger distances that the BioNano technology spanned while joining scaffolds (described above) might make it less likely that two joined scaffolds fall into a region that is syntenic with A17 given that synteny tends to decrease with distance. BioNano-joined scaffolds, therefore, might map to multiple A17 chromosomes more than Dovetail-joined scaffolds due to synteny breakdown rather than inaccuracy of joins. However, given that BioNano gaps span less than $200 \mathrm{~kb}$ and that the majority of the R108 genome has synteny blocks with A17 that are greater than $1 \mathrm{Mb}$ (Figs. 1, 2, 3) [39], we expect this different to be small and the difference between Dovetail and BioNano join accuracy to be real.

Alternatively, Dovetail breaks performed much worse than joins using A17 synteny as a measure. Of the $\mathrm{PbBn}$ scaffolds subsequently broken by Dovetail in the $\mathrm{PbBnDt}$ assembly, only $40 \%$ of them mapped to different A17 chromosomes, indicating that Dovetail might be breaking more correct BioNano joins than incorrect ones.

A17 chromosomal mapping is far from a perfect gold standard given the evolutionary distance between A17 and R108. Joined segments of R108 scaffolds that map to different A17 chromosomes may still map to the same R108 chromosome. Indeed, one of the joins shared by both Dovetail and BioNano that mapped to different A17 chromosomes corresponds to the known chromosome 4/8 translocation. This join, therefore, is correct, even though synteny to A17 put it on two different chromosomes. It is possible that there are other regions where synteny to A17 doesn't accurately predict synteny in R108. Using long-range physical information, such as $\mathrm{Hi}-\mathrm{C}$ data or a genetic map that involves R108, could allow us to better validate the BioNano and Dovetail technologies as well as to obtain chromosome-scale ordering of the genome assembly.

\section{Strengths and weaknesses dictate strategy for ordering technologies}

For the final assembly, we chose to gap-fill the PbDtBn assembly rather than the PbBnDt assembly. This decision was based not only on comparisons of important assembly continuity and completeness statistics, as described above, but also on the knowledge we uncovered about the differences between the scaffolding and mapping technologies.

One important difference between the two technologies is their ability to incorporate smaller scaffolds. In our study, Dovetail incorporated thirty-five small scaffolds (less than $100 \mathrm{~kb}$ ) over both rounds but BioNano incorporated only one. The minimum scaffold size joined by BioNano $(98.1 \mathrm{~kb})$ was more than 20 times larger than the minimum scaffold size joined by Dovetail $(4.8 \mathrm{~kb})$. Similar results were found when applying BioNano maps to the short arm of wheat chromosome 7D where the optimum size for incorporation by BioNano was $90 \mathrm{~kb}$ or higher [56] and sequences shorter than $30 \mathrm{~kb}$ could not anchored reliably. Given that the scaffold N50 was $3.7 \mathrm{Mb}$ in the $\mathrm{Pb}$ assembly to which these technologies was added, the discrepancy between the two technologies in joining scaffolds less than $100 \mathrm{~kb}$ did not have as great an effect on our assemblies. However, if a much more fragmented assembly were used, we would expect Dovetail to perform much better than BioNano if only one scaffolding or mapping technology were used. If both technologies are used, applying Dovetail first to incorporate the smaller scaffolds and create a more contiguous substrate for BioNano to use makes sense and would be especially critical for highly fragmented assemblies.

A second difference in the two technologies also supports applying Dovetail prior to BioNano for combined strategies. Dovetail breaks sequences it identifies as chimeric as it runs the software. BioNano logs potential chimeric sequences, but does not induce breaks in the assembly without manual intervention. Hence, if BioNano is applied first, chimeric contigs may not yet be properly separated when the assembler's master plan for scaffolding is being formed. Having a more accurate assembly up-front, as should occur when Dovetail is applied first, is always best before scaffolding assemblies.

Both technologies were able to bridge larger duplicated and/or repetitive regions than was PacBio, which requires multiple reads long enough to span an ambiguous region. With only $10 \%$ of the sequenced nts in PacBio reads longer than 18,555 nt (N10), the ability of PacBio to span ambiguous regions is likely limited to a similar size, though longer reads will increase the size of the spannable repeats. Therefore, both mapping technologies can add value for spanning ambiguous regions that are beyond the reach of current PacBio capabilities. However, both technologies are limited in the size of gap they can span. Dovetail is limited by its longest pairs, which in this study, likely kept joins to around $100 \mathrm{~kb}$ or less, though without sized gaps it is difficult to figure out the true maximum. BioNano can join scaffolds over much larger gaps. The largest span made in this study created a gap of nearly $650 \mathrm{~kb}$, though most joins spanned less than $100 \mathrm{~kb}$ (Table 3). Nevertheless, Dovetail and BioNano both were able to span ambiguous regions that were beyond PacBio's current capability. 


\section{Conclusions}

The use and analysis of both BioNano and Dovetail technologies in all possible combinations is novel and yielded strategic information about how best to apply these strategies to PacBio. Both technologies were able to span repetitive regions that PacBio was unable to bridge. Using PacBio, followed by Dovetail and then BioNano, and then gap-filled with PBJelly, we have generated a second, reference quality assembly for $M$. truncatula. Because of the distance between R108 and the A17 reference as well as the inability to interbreed them to create a genetic map, having a second high quality $M$. truncatula reference has been a priority in the Medicago truncatula community. A second reference assembly has yielded novel sequence and will be an important resource for the R108 functional community to support gene-finding in the Tnt1 lines. The R108 reference assembly has also allowed us to investigate the details of the A17 translocation.

\section{Methods}

We generated five genome assemblies: a PacBio only assembly $(\mathrm{Pb})$, a PacBio base assembly that was scaffold together with either Dovetail (PbDt) or BioNano (PtBn), a $\mathrm{Pb}$ base assembly that was scaffold together with Dovetail and then BioNano (PbDtBn) and a Pb base assembly that was scaffold together with BioNano and then Dovetail (PbBnDt). The completeness of each assembly was evaluated by alignments of PacBio reads as well as independent Illumina reads, and capture of an independent transcriptome as well as core eukaryotic genes. For comparison, we used the A17 version 4.0 reference genome [44].

\section{PacBio sequencing and assembly}

DNA for PacBio assemblies was obtained from fifty grams of young leaf tissue obtained from multiple plants grown in the greenhouse and dark-treated for $24 \mathrm{~h}$. High molecular weight genomic DNA was generated by Amplicon Express (Pullman, WA) using their standard BAC nuclei prep followed by a CTAB liquid DNA precipitation.

Whole-genome DNA sequencing was performed using a Pacific Biosciences RS II instrument (Pacific BioSciences, Menlo Park, CA). Libraries were constructed using the PacBio 20-Kb protocol [59]. These libraries were loaded onto 122 SMRT cells and sequenced using P4/P6 polymerase and C2/C4 chemistry with 3- and 6-h movie times, respectively. PacBio sequencing yielded approximately $107 \mathrm{X}$ sequence coverage. A de novo assembly of PacBio reads was generated using FALCON [20] assembler version 0.4 using default parameters. Contigs smaller than $1 \mathrm{~kb}$ were removed. In order to improve the accuracy of the assembly, Quiver polishing was done on SMRT portal (version smrtanalysis_2.3.0.140936.p5. 167094) using the "RS_Resequencing" protocol using the latest version available at the time.

\section{Dovetail}

DNA from Amplicon Express (described above) was used. A Chicago library (Dovetail Genomics LLC, Santa Cruz, CA) [18] was generated using the DpnII restriction endonuclease (GATC). Briefly, this entailed reconstituting chromatin using purified histones and chromatin assembly factors, followed by cross-linking the chromatin using formaldehyde. DNA was then digested using the DpnII restriction endonuclease. The resulting sticky ends were filled in with thiolated and biotinylated nucleotides. A blunt end ligation of free ends followed by removal of the crosslinking and proteins yielded fragments with DNA joined across distances of up to about $100 \mathrm{~kb}$. An exonuclease was used to remove the biotinylated nucleotides. The thiolated nucleotides, which were proximal to the biotinylated nucleotides, protected the DNA from further exonucleation.

The resulting DNA fragments were taken through a standard Illumina library prep, including shearing and adapter ligation. The library was sequenced on an Illumina HiSeq $2000(2 \times 100$ Base Pairs $)$ to a physical coverage level of $\sim 588 \mathrm{X}$ ( $67 \mathrm{X}$ sequence coverage).

Sequence data generated from this library were used to scaffold the PacBio de novo assembly through Dovetail's HiRise ${ }^{\mathrm{Tx}}$ pipeline v. 1.3.0-57-g4d1fc9b [18]. In short, Chicago library reads were mapped back to the assembly using a modified version of SNAP (http://snap.cs.berkeley.edu/). Pairs in which both reads were uniquely mapped were used to generate a likelihood model representing how chromatin crosslinking brings sequences together. A graph where the nodes are contigs and the edges are ordered integer pairs representing placement of the paired reads in the contigs was used for scaffolding beginning with high confidence linear subpaths and prioritizing joins in order of log likelihood improvement. During the process, in addition to joining sequences, putative chimeric sequences were broken. An iterative approach was taken by feeding the resulting scaffolds back into the pipeline. Refinement of local ordering and orientation and gap closing using Meraculous's Marauder module was done at the end [60].

\section{BioNano}

Five grams of young leaf tissue was obtained from greenhouse-grown plants dark-treated for $24 \mathrm{~h}$ before harvest. High molecular weight DNA was extracted and a de novo whole genome map assembly was generated using the BioNano Genomics (BNG) (BioNano Genomics, San Diego, CA) platform at the Bioinformatics Center at Kansas State University. High Molecular Weight 
(HMW) DNA was nicked and labeled according to the IrysPrep protocol. In brief, HMW DNA was double digested by a cocktail of single-stranded nicking endonucleases, Nt.BspQI (GCTCTTC) and Nt.BbvCI (CCTC AGC), and then labeled with a fluorescent-dUTP nucleotide analog using Taq polymerase. Nicks were ligated with Taq DNA ligase and the backbone of the labeled DNA was stained using the intercalating dye, YOYO-1. The nicked and labeled DNA was then loaded onto an IrysChip for imaging automatically on the Irys system (BioNano Genomics). BNG molecules were filtered with a minimum length of $150 \mathrm{~kb}$ and 8 minimum labels. A $p$-value threshold for the BNG assembler was set to a minimum of 2.6e-9. Molecules were assembled with BioNano Pipeline Version 2884 and RefAligner Version 2816 [55].

For BioNano scaffolding, hybridScaffold.pl version 4618 from BioNano Genomics was used. The input assembly fasta sequence was nicked in silico for Nt.BspQI and Nt.BbvCI labels. Consensus Maps (CMAP) were only created for scaffolds $>20 \mathrm{kbp}$ with $>5$ labels. A $p$-value of $1 e-10$ was used as a minimum confidence value to output initial (BNG consensus map to in silico cmap). The final (in silico cmap to final hybrid cmap) alignments and a $p$-value of $1 e-13$ were used as minimum confidence value to flag chimeric/conflicting alignments and to merge alignments. Scaffolds that were not super-scaffolded were added to the output from hybridScaffold.pl.

The BNG scaffolding pipeline identifies potential breaks that should be made to the base assembly in the form of a chimera file, but these suggested breaks are not made without manual intervention. We did not attempt to make any of the BioNano breaks. For BioNano joins, only joins that incorporated more than one scaffold were considered.

BioNano sizes gaps but does not fill them exclusively with Ns. Rather, BioNano adds in restriction site recognition sequences within the gap according to where restriction sites were seen in the BioNano map. This results in hundreds of tiny contigs which break up the BioNano gaps into smaller fragments. For the purposes of this paper, we used the GAEMR basic stats default of using 200 as a minimum contig size, effectively ignoring these restriction sites island for calculating assembly statistics and obtaining a single gap per join.

\section{Illumina}

In order to compare the completeness of assemblies constructed with different combinations of PacBio, Dovetail, and BioNano, we collected Illumina data that was independent of the assemblies. Illumina short-insert paired ends were generated from an independent DNA sample using TrueSeq v3.0 chemistry and sequenced on an Illumina $\mathrm{HiSeq}^{\circ}$ 2000. A total of $332,236,248$ reads (71.4X coverage) of length $100 \mathrm{nt}$ were generated.

\section{Transcriptome assembly}

To evaluate how the transcriptome was represented in the genome assemblies, the transcriptome of 14 day old R108 roots was sequenced using Illumina's RNA-Seq protocol. The transcriptome was assembled using the Transcriptome Assembly Pipeline (BPA2.1.0) [61]. The BPA pipeline includes a kmer sweep assembly strategy with ABySS (using the kmer values of 50, 60, 70, 80 and 90) [62], followed by an OLC (overlap layout consensus) assembly with CAP3 [63] to find overlaps between contigs (unitigs). Scaffolding with ABySS and gap closure were performed to obtain the final assembled transcriptome sequences (Simpson et al. 2009). The transcripts were clustered at $98 \%$ sequence identity using the CDHIT-EST software [64]. Finally, the set of transcript sequences were filtered by length (minimum length of $100 \mathrm{bp}$ ). An additional filtering step using ESTScan [65] was performed to identify open reading frames using $M$. truncatula protein coding genes as a reference, yielding the final transcriptome set. Transcripts were mapped against each of the five assemblies using GMAP [66]. Transcript hits were retained if aligning along at least $90 \%$ of their sequence with at least $90 \%$ identity.

\section{BUSCO}

Benchmarking Universal Single Copy Orthologs (BUSCO) provides a quantitative assessment of genome assemblies based on orthologs selected from OrthoDB [47]. Assembly assessments were performed using the plant early release of BUSCO v1.1b1, which contains 956 genes that are present in at least $90 \%$ of the plant species used to assemble the database [47]. tBLASTn searches were used to identify BUSCOs followed by Augustus gene predictions and classified into lineage specific matches using HMMER within the BUSCO package.

\section{Read alignments}

In order to assess the completeness of the assembly, PacBio filtered (minimum length of 50 and minimum quality of 75) subreads were realigned to the five assemblies using the BLASR mapper [67]. All the subreads were considered for the alignment to the assemblies (-useallccs). Illumina reads were aligned to the five assemblies using the Burrows-Wheeler Aligner (BWA), version 0.7.12 with a maximum of 2 paths and sam output format.

\section{Structural annotation}

To understand how gene sequences were affected by the assembly strategies, the MAKER-P genome annotation pipeline was used to annotate the five genome 
assemblies [68-70]. All available M. truncatula R108 transcripts were assembled using the Trinity Assembler. All transcripts were from a single tissue, root, which is not ideal. Nevertheless, GMAP alignments to A17 indicate that the transcript assembly contains the majority of genes. Further, within the five assemblies, relative capture rates of these transcripts should not be biased by the lack of evidence transcripts from multiple tissues.

The resulting assembly was used as input for expressed sequence tag (EST) evidence for MAKER-P annotations [71, 72]. The MAKER-P pipeline aligns the provided ESTs to the genome and creates ab initio gene predictions with SNAP [73] and Augustus [74, 75] using evidence-based quality values. Each assembly was divided into ten chunks and processed through MAKER-P individually. Following completion of MAKER-P runs for each of the ten chunks, fasta and gff files were combined using fasta_merge and gff3_merge, respectively, included as part of the MAKER-P package.

\section{Identification of structural rearrangements and novel sequences in R108}

Each R108 PacBio-based assembly was first aligned to the A17 reference (i.e., Mt4.0) using BLAT [76]. The resulting alignments were merged, fixed (removing nonsyntenic or overlapping alignment blocks) and cleaned (removing alignment blocks containing assembly gaps). BLAT Chain/Net tools were then used to obtain a single coverage best alignment net in the target genome (HM101) as well as a reciprocal-best alignment net between genomes. Finally, genome-wide synteny blocks were built for each assembly (against HM101), enabling identification of genome structural rearrangements including the chr4-8 translocation.

Based on pairwise genome comparison of R108 and A17, we obtained a raw set of novel sequences (present in R108 but absent in A17) by subtracting all aligned regions from the gap-removed assembly. Lowcomplexity sequences and short tandem repeats were scanned and removed using Dustmasker [77] and Tandem Repeat Finder [78]. Potential contaminant sequences (best hit in non-plant species) were filtered by BLASTing [79] against NCBI Nucleotide (nr/nt) database. Genes with more than 50\% CDS in these regions comprised the accession-specific gene set. Pfam analysis and functional enrichment were then performed on this novel gene list [80].

\section{Additional files}

Additional file1: Supplementary Figures and Tables. Contains Supplementary Figure S1 and Supplementary Tables S1-S5. (DOCX $110 \mathrm{~kb})$

\section{Abbreviations}

Bn: BioNano; Dt: Dovetail; Pb: PacBio; PbBn: PacBio BioNano; PbBnDt: PacBio BioNano Dovetail; PbDt: PacBio Dovetail; PbDtBn: PacBio Dovetail BioNano

\section{Acknowledgements}

We thank Roxanne Denny (UMN) for generating plant material, Susan Brown, Michelle Coleman and Jennifer Shelton from Kansas State University for the BioNano Maps, and Margot Hartley at Dovetail for generating the Dovetail libraries and running HiRise.

\section{Funding}

This research was funded by National Science Foundation Grant 1,237,993 to NDY, PT, KATS, RMS, JRM and JM. The funding source was not involved in the design of the study, collection, analysis, interpretation of data, or in the writing of the manuscript.

\section{Availability of data and materials}

The R108 v1.0 assembly, sample information and the raw PacBio reads are available in Genbank (BioProject: PRJNA368719, Biosample: SAMN04571790, PacBio reads: SRS1353205, assembly MWMB00000000.1). The gene annotation (GFF3) and BioNano (BNX) files are available under DOI numbers DOI: 10.13140/ RG.2.2.28253.51686 and DOI: 10.13140/RG.2.2.32950.80964, respectively. The R108 RNA-Seq data are available in the NCBI sequence read archive (SRA), under BioProject accession number SRP077692.

\section{Authors' contributions}

JM, KATS, NDY, JRM, and PT conceived and designed this research. KMM, PZ, TR, DF, NPD, and JM did the assemblies and analyses. MJS and RMS generated the R108 RNA-Seq data. JM, KMM, PZ, TR, and DF wrote the manuscript. KATS, NDY, JRM, PT, RMS, and MJS edited the manuscript. All authors read and approved the final manuscript.

\section{Ethics approval and consent to participate}

Medicago germplasm resources (seed) were obtained and used, with permission, from Jean-Marie Prosperi at Unité mixte de recherche / Amélioration génétique et adaptation des plantes méditerranéennes et tropicales (UMR-AGAP) at INRAMontpellier, France.

\section{Consent for publication \\ Not applicable.}

\section{Competing interests}

The authors declare that they have no competing interests.

\section{Publisher's Note}

Springer Nature remains neutral with regard to jurisdictional claims in published maps and institutional affiliations.

\section{Author details}

${ }^{1}$ National Center for Genome Resources, 2935 Rodeo Park Drive East, Santa Fe, NM 87505, USA. ${ }^{2}$ Montana State University, Center for Biofilm Engineering, Bozeman, MT 59717, USA. ${ }^{3}$ Department of Plant Biology, University of Minnesota, Saint Paul, MN, USA. ${ }^{4}$ Department of Soil, Water \& Climate, Plant and Microbial Biology and BioTechnology Institute, University of Minnesota, St. Paul, MN, USA. ${ }^{5}$ Department of Agronomy and Plant Genetics, University of Minnesota, Saint Paul, MN, USA. ${ }^{6}$ Department of Plant and Microbial Biology, University of Minnesota, Saint Paul, MN, USA. ${ }^{7}$ J. Craig Venter Institute, Rockville, MD, USA. ${ }^{8}$ Minnesota Supercomputing Institute, University of Minnesota, Minneapolis, MN, USA.

Received: 4 May 2017 Accepted: 31 July 2017

Published online: 04 August 2017

References

1. Metzker ML. Sequencing technologies - the next generation. Nat Rev Genet. 2010;11(1):31-46.

2. Shendure J, Lieberman Aiden E. The expanding scope of DNA sequencing. Nat Biotechnol. 2012;30(11):1084-94.

3. Mostovoy Y, Levy-Sakin M, Lam J, Lam ET, Hastie AR, Marks P, Lee J, Chu C, Lin C, Džakula Ž. A hybrid approach for de novo human genome sequence assembly and phasing. Nat Methods. 2016;13(7):587-90. 
4. VanBuren R, Bryant D, Edger PP, Tang H, Burgess D, Challabathula D, Spittle K, Hall R, Gu J, Lyons E. Single-molecule sequencing of the desiccationtolerant grass Oropetium thomaeum. Nature. 2015;527(7579):508-11.

5. Pendleton M, Sebra R, Pang AW, Ummat A, Franzen O, Rausch T, Stutz AM, Stedman W, Anantharaman T, Hastie A, et al. Assembly and diploid architecture of an individual human genome via single-molecule technologies. Nat Methods. 2015;12(8):780-6.

6. Jiao W-B, Accinelli GG, Hartwig B, Kiefer C, Baker D, Severing E, Willing E-M, Piednoel M, Woetzel S, Madrid-Herrero E. Improving and correcting the contiguity of long-read genome assemblies of three plant species using optical mapping and chromosome conformation capture data. Genome Res. 2017;27(5):778-86

7. Jiao Y, Peluso P, Shi J, Liang T, Stitzer MC, Wang B, Campbell MS, Stein JC, Wei $X$, Chin C-S. Improved maize reference genome with single-molecule technologies. Nature. 2017;

8. Bickhart DM, Rosen BD, Koren S, Sayre BL, Hastie AR, Chan S, Lee J, Lam ET, Liachko I, Sullivan ST. Single-molecule sequencing and conformational capture enable de novo mammalian reference genomes. bioRxiv. 2016;1:064352.

9. Ashrafi H. Using spinach to compare technologies for whole genome assemblies, In: Plant and Animal Genome Conference XXIII 2015. San Diego, CA; 2015.

10. Bertioli DJ, Cannon SB, Froenicke L, Huang G, Farmer AD, Cannon EK, Liu X, Gao D, Clevenger J, Dash S, et al. The genome sequences of Arachis duranensis and Arachis ipaensis, the diploid ancestors of cultivated peanut. Nat Genet. 2016;48(4):438-46.

11. Lam ET, Hastie A, Lin C, Ehrlich D, Das SK, Austin MD, Deshpande P, Cao $H$, Nagarajan N, Xiao M, et al. Genome mapping on nanochannel arrays for structural variation analysis and sequence assembly. Nat Biotechnol. 2012;30(8):771-6.

12. Chaney L, Sharp AR, Evans CR, Udall JA. Genome Mapping in Plant Comparative Genomics. Trends Plant Sci. 2016;21(9):770-80.

13. Schwartz DC, Li X, Hernandez LI, Ramnarain SP, Huff EJ, Wang Y-K. Ordered restriction maps of Saccharomyces Cerevisiae chromosomes constructed by optical mapping. Science. 1993;262(5130):110-4.

14. Imelfort M, Edwards D. De novo sequencing of plant genomes using second-generation technologies. Brief Bioinform. 2009;10(6):609-18.

15. Somes K, Das MDA, Matthew C, Akana PD, Cao H, Xiao M. Single molecule linear analysis of DNA in nano-channel labeled with sequence specific fluorescent probes. Nucleic Acids Res. 2010;38(18):1-8.

16. Shelton JM, Coleman MC, Herndon N, Lu N, Lam ET, Anantharaman T, Sheth P, Brown SJ. Tools and pipelines for BioNano data: molecule assembly pipeline and FASTA super scaffolding tool. BMC Genomics. 2015;16(1):734.

17. Belton JM, McCord RP, Gibcus JH, Naumova N, Zhan Y, Dekker J. Hi-C: a comprehensive technique to capture the conformation of genomes. Methods. 2012;58(3):268-76.

18. Putnam NH, O'Connell BL, Stites JC, Rice BJ, Blanchette M, Calef R, Troll CJ, Fields A, Hartley PD, Sugnet CW et al. Chromosome-scale shotgun assembly using an in vitro method for long-range linkage. Genome Res. 2016.

19. Schatz MC, Witkowski J, McCombie WR. Current challenges in de novo plant genome sequencing and assembly. Genome Biol. 2012;13(4):1.

20. Chin C-S, Peluso P, Sedlazeck FJ, Nattestad M, Concepcion GT, Clum A, Dunn C, O'Malley R, Figueroa-Balderas R, Morales-Cruz A, et al. Phased diploid genome assembly with single-molecule real-time sequencing. Nat Meth. 2016;13(12):1050-4.

21. Jarvis DE, Ho YS, Lightfoot DJ, Schmöckel SM, Li B, Borm TJ, Ohyanagi $H$, Mineta K, Michell CT, Saber N. The genome of Chenopodium Quinoa. Nature. 2017;542(7641):307-12.

22. Zapata L, Ding J, Willing E-M, Hartwig B, Bezdan D, Jiao W-B, Patel V, James GV, Koornneef M, Ossowski S. Chromosome-level assembly of Arabidopsis thaliana Ler reveals the extent of translocation and inversion polymorphisms. Proc Natl Acad Sci. 2016;27:201607532.

23. Berlin K, Koren S, Chin C-S, Drake JP, Landolin JM, Phillippy AM. Assembling large genomes with single-molecule sequencing and locality-sensitive hashing. Nat Biotechnol. 2015;33(6):623-30.

24. Zhang J, Chen L-L, Xing F, Kudrna DA, Yao W, Copetti D, Mu T, Li W, Song $J-M, X i e$ W. Extensive sequence divergence between the reference genomes of two elite indica rice varieties Zhenshan 97 and Minghui 63. Proc Natl Acad Sci. 2016;113(35):E5163-71.

25. Daccord N, Celton J-M, Linsmith G, Becker C, Choisne N, Schijlen E, van de Geest H, Bianco L, Micheletti D, Velasco R. High-quality de novo assembly of the apple genome and methylome dynamics of early fruit development. Nat Genet. 2017;49(7):1099-106.
26. Du H, Yu Y, Ma Y, Gao Q, Cao Y, Chen Z, Ma B, Qi M, Li Y, Zhao X. Sequencing and de novo assembly of a near complete indica rice genome. Nat Commun. 2017;8:15324.

27. Reyes-Chin-Wo S, Wang Z, Yang X, Kozik A, Arikit S, Song C, Xia L, Froenicke L, Lavelle DO, Truco M-J. Genome assembly with in vitro proximity ligation data and whole-genome triplication in lettuce. Nat Commun. 2017:8:14953.

28. Bredeson JV, Lyons JB, Prochnik SE, Wu GA, Ha CM, Edsinger-Gonzales E, Grimwood J, Schmutz J, Rabbi IY, Egesi C. Sequencing wild and cultivated cassava and related species reveals extensive interspecific hybridization and genetic diversity. Nat Biotechnol. 2016;34(5):562-70.

29. Pootakham W, Sonthirod C, Naktang C, Ruang-Areerate P, Yoocha T, Sangsrakru D, Theerawattanasuk K, Rattanawong R, Lekawipat N, Tangphatsornruang S. De novo hybrid assembly of the rubber tree genome reveals evidence of paleotetraploidy in Hevea species. Sci Rep. 2017;7:41457.

30. Sato S, Nakamura Y, Kaneko T, Asamizu E, Kato T, Nakao M, Sasamoto S, Watanabe A, Ono A, Kawashima K. Genome structure of the legume, Lotus Japonicus. DNA Res. 2008:15(4):227-39.

31. Schmutz J, Cannon SB, Schlueter J, Ma J, Mitros T, Nelson W, Hyten DL, Song Q, Thelen JJ, Cheng J, et al. Genome sequence of the palaeopolyploid soybean. Nature. 2010;463(7278):178-83.

32. Young ND, Debelle F, Oldroyd GE, Geurts R, Cannon SB, Udvardi MK, Benedito VA, Mayer KF, Gouzy J, Schoof H, et al. The Medicago genome provides insight into the evolution of rhizobial symbioses. Nature. 2011; 480(7378):520-4.

33. Varshney RK, Song C, Saxena RK, Azam S, Yu S, Sharpe AG, Cannon S, Baek J, Rosen BD, Tar'an B, et al. Draft genome sequence of chickpea (Cicer Arietinum) provides a resource for trait improvement. Nat Biotechnol. 2013;31(3):240-6.

34. Kang YJ, Kim SK, Kim MY, Lestari P, Kim KH, Ha BK, Jun TH, Hwang WJ, Lee $\mathrm{T}$, Lee J, et al. Genome sequence of mungbean and insights into evolution within Vigna species. Nat Commun. 2014;5:5443.

35. Chen X, Li H, Pandey MK, Yang Q, Wang X, Garg V, Li H, Chi X, Doddamani $D$, Hong $Y$, et al. Draft genome of the peanut A-genome progenitor (Arachis duranensis) provides insights into geocarpy, oil biosynthesis, and allergens. Proc Natl Acad Sci U S A. 2016;113(24):6785-90.

36. Li YH, Zhou G, Ma J, Jiang W, Jin LG, Zhang Z, Guo Y, Zhang J, Sui Y, Zheng $L$, et al. De novo assembly of soybean wild relatives for pan-genome analysis of diversity and agronomic traits. Nat Biotechnol. 2014;32(10):1045-52.

37. Gan X, Stegle O, Behr J, Steffen JG, Drewe P, Hildebrand KL, Lyngsoe R, Schultheiss SJ, Osborne EJ, Sreedharan VT, et al. Multiple reference genomes and transcriptomes for Arabidopsis Thaliana. Nature. 2011:477(7365):419-23.

38. Schatz MC, Maron LG, Stein JC, Wences AH, Gurtowski J, Biggers E, Lee H, Kramer M, Antoniou E, Ghiban E. Whole genome de novo assemblies of three divergent strains of rice, Oryza Sativa, document novel gene space of aus and indica. Genome Biol. 2014;15(11):1.

39. Zhou P, Silverstein KA, Ramaraj T, Guhlin J, Denny R, Liu J, Farmer AD, Steele KP, Stupar RM, Miller JR. Exploring structural variation and gene family architecture with de novo assemblies of 15 Medicago genomes. BMC Genomics. 2017;18(1):261.

40. Golicz AA, Bayer PE, Barker GC, Edger PP, Kim H, Martinez PA, Chan CKK, Severn-Ellis A, McCombie WR, Parkin IA. The pangenome of an agronomically important crop plant Brassica Oleracea. Nat Commun. 2016;7:13390.

41. Branca A, Paape TD, Zhou P, Briskine R, Farmer AD, Mudge J, Bharti AK, Woodward JE, May GD, Gentzbittel L. Whole-genome nucleotide diversity, recombination, and linkage disequilibrium in the model legume Medicago Truncatula. Proc Natl Acad Sci. 2011;108(42):E864-70.

42. Tadege M, Ratet P, Mysore KS. Insertional mutagenesis: a Swiss Army knife for functional genomics of Medicago Truncatula. Trends Plant Sci. 2005;10(5):229-35.

43. Tadege M, Wen J, He J, Tu H, Kwak Y, Eschstruth A, Cayrel A, Endre G, Zhao PX, Chabaud M, et al. Large-scale insertional mutagenesis using the Tnt1 retrotransposon in the model legume Medicago Truncatula. Plant J. 2008; 54(2):335-47.

44. Tang H, Krishnakumar V, Bidwell S, Rosen B, Chan A, Zhou S, Gentzbittel L, Childs KL, Yandell M, Gundlach H. An improved genome release (version Mt4. 0) for the model legume Medicago Truncatula. BMC Genomics. 2014;15(1):1.

45. Steven B, Cannon LS, Rombauts S, Sato S, Cheung F, Gouzy J, Wang X, Mudge J, Vasdewani J, Schiex T, Spannagl M, Monaghan E, Nicholson C, Humphray SJ, Schoof H, Mayer KFX, Rogers J, Quétier F, Oldroyd GE, Debellé F, Cook DR, Retzel EF, Roe BA, Town CD, Tabata S, de Peer YV, Young ND. Legume genome evolution viewed through the Medicago Truncatula and Lotus Japonicus genomes. PNAS. 2003;103(40):14959-64. 
46. Blanc $\mathrm{G}$, Wolfe KH. Widespread paleopolyploidy in model plant species inferred from age distributions of duplicate genes. Plant Cell. 2004;16(7):1667-78.

47. Simao FA, Waterhouse RM, loannidis P, Kriventseva EV, Zdobnov EM. BUSCO: assessing genome assembly and annotation completeness with single-copy orthologs. Bioinformatics. 2015;31(19):3210-2.

48. Gnerre S, MacCallum I, Przybylski D, Ribeiro FJ, Burton JN, Walker BJ, Sharpe T, Hall G, Shea TP, Sykes S. High-quality draft assemblies of mammalian genomes from massively parallel sequence data. Proc Natl Acad Sci. 2011;108(4):1513-8.

49. Kamphuis LG, Williams AH, D'Souza NK, Pfaff T, Ellwood SR, Groves EJ, Singh KB, Oliver RP, Lichtenzveig J. The Medicago Truncatula reference accession A17 has an aberrant chromosomal configuration. New Phytol. 2007;174(2):299-303.

50. Chin C-S, Alexander DH, Marks P, Klammer AA, Drake J, Heiner C, Clum A, Copeland A, Huddleston J, Eichler EE. Nonhybrid, finished microbial genome assemblies from long-read SMRT sequencing data. Nat Methods. 2013;10(6):563-9.

51. Koren S, Schatz MC, Walenz BP, Martin J, Howard JT, Ganapathy G, Wang Z, Rasko DA, McCombie WR, Jarvis ED. Hybrid error correction and de novo assembly of single-molecule sequencing reads. Nat Biotechnol. 2012;30(7):693-700

52. Koren S, Phillippy AM. One chromosome, one contig: complete microbial genomes from long-read sequencing and assembly. Curr Opin Microbiol. 2015;23:110-20.

53. Ribeiro FJ, Przybylski D, Yin S, Sharpe T, Gnerre S, Abouelleil A, Berlin AM, Montmayeur A, Shea TP, Walker BJ. Finished bacterial genomes from shotgun sequence data. Genome Res. 2012;22(11):2270-7.

54. English AC, Richards S, Han Y, Wang M, Vee V, Qu J, Qin X, Muzny DM, Reid JG, Worley KC. Mind the gap: upgrading genomes with Pacific Biosciences RS long-read sequencing technology. PLoS One. 2012;7(11):e47768.

55. Hongzhi Cao ARH, Cao D, Lam ET, Sun Y, Huang H, Liu X, Lin L, Andrew W, Chan S, Huang S, Tong X, Requa M, Anantharaman T, Krogh A, Yang H, $\mathrm{Cao} H, \mathrm{Xu}$ X. Rapid detection of structural variation in a human genome using nanochannel-based genome mapping technology. GigaScience. 2014;3(34):1-11.

56. Stankova H, Hastie AR, Chan S, Vrana J, Tulpova Z, Kubalakova M, Visendi P, Hayashi S, Luo M, Batley J, et al. BioNano genome mapping of individual chromosomes supports physical mapping and sequence assembly in complex plant genomes. Plant Biotechnol J. 2016;14(7):1523-31.

57. Meyer CA, Liu XS. Identifying and mitigating bias in next-generation sequencing methods for chromatin biology. Nat Rev Genet. 2014;15(11):709-21.

58. Yaffe $\mathrm{E}$, Tanay A. Probabilistic modeling of hi-C contact maps eliminates systematic biases to characterize global chromosomal architecture. Nat Genet. 2011;43(11):1059-65.

59. Kim KE, Peluso P, Babayan P, Yeadon PJ, Yu C, Fisher WW, Chin CS, Rapicavoli NA, Rank DR, Li J, et al. Long-read, whole-genome shotgun sequence data for five model organisms. Sci Data. 2014;1:140045.

60. Chapman JA, Ho I, Sunkara S, Luo S, Schroth GP, Rokhsar DS. Meraculous: de novo genome assembly with short paired-end reads. PLoS One. 2011;6(8):e23501.

61. Santoferrara LF, Guida S, Zhang H, McManus GB. De novo transcriptomes of a mixotrophic and a heterotrophic ciliate from marine plankton. PLoS One. 2014;9(7):e101418.

62. Simpson JT, Wong K, Jackman SD, Schein JE, Jones SJ, Birol I. ABySS: a parallel assembler for short read sequence data. Genome Res. 2009;19(6):1117-23.

63. Huang X, Madan A. CAP3: a DNA sequence assembly program. Genome Res. 1999:9(9):868-77.

64. Li W, Godzik A. Cd-hit: a fast program for clustering and comparing large sets of protein or nucleotide sequences. Bioinformatics. 2006;22(13):1658-9.

65. Iseli C, Jongeneel CV, Bucher P. ESTScan a program for detecting, evaluating, and reconstructing potential coding regions in ESTsequences. SMB-99 Proceedings. 1999;99:138-48.

66. Wu TD, Watanabe CK. GMAP: a genomic mapping and alignment program for mRNA and EST sequences. Bioinformatics. 2005;21(9):1859-75.

67. Chaisson MJ, Tesler G. Mapping single molecule sequencing reads using basic local alignment with successive refinement (BLASR) application and theory. BMC Bioinformatics. 2012;13(328):1-17.

68. Holt C, Yandell M. MAKER2: an annotation pipeline and genome-database management tool for second-generation genome projects. BMC Bioinformatics. 2011;12:491

69. Cantarel BL, Korf I, Robb SMC, Parra G, Ross E, Moore B, Holt C, Sánchez Alvarado A, Yandell M. MAKER: an easy-to-use annotation pipeline designed for emerging model organism genomes. Genome Res. 2008;18(1):188-96.
70. Campbell MS, Holt C, Moore B, Yandell M. Genome annotation and Curation using MAKER and MAKER-P. Curr Protoc Bioinformatics. 2014;48:4 11 11-14 1139.

71. Grabherr MG, Haas BJ, Yassour M, Levin JZ, Thompson DA, Amit I, Adiconis $X$, Fan L, Raychowdhury R, Zeng Q, et al. Full-length transcriptome assembly from RNA-Seq data without a reference genome. Nat Biotechnol. 2011;29(7):644-52.

72. Haas BJ, Papanicolaou A, Yassour M, Grabherr M, Blood PD, Bowden J, Couger $M B$, Eccles D, Li B, Lieber M, et al. De novo transcript sequence reconstruction from RNA-seq using the trinity platform for reference generation and analysis. Nat Protoc. 2013;8(8):1494-512.

73. Korf I. Gene finding in novel genomes. Bmc Bioinformatics. 2004;5(1):59.

74. Stanke M, Waack S. Gene prediction with a hidden Markov model and a new intron submodel. Bioinformatics. 2003;19(Suppl 2):ii215-25.

75. Stanke M, Keller O, Gunduz I, Hayes A, Waack S, Morgenstern B. AUGUSTUS: ab initio prediction of alternative transcripts. Nucleic Acids Res. 2006;34(Web Server issue):W435-9.

76. Kent WJ. BLAT-the BLAST-like alignment tool. Genome Res. 2002;12(4):656-64

77. Morgulis A, Gertz EM, Schäffer AA, Agarwala R. A fast and symmetric DUST implementation to mask low-complexity DNA sequences. J Comput Biol. 2006;13(5):1028-40.

78. Benson G. Tandem repeats finder: a program to analyze DNA sequences. Nucleic Acids Res. 1999:27(2):573.

79. Altschul SF, Gish W, Miller W, Myers EW, Lipman DJ. Basic local alignment search tool. J Mol Biol. 1990;215(3):403-10.

80. Finn RD, Coggill P, Eberhardt RY, Eddy SR, Mistry J, Mitchell AL, Potter SC, Punta M, Qureshi M, Sangrador-Vegas A. The Pfam protein families database: towards a more sustainable future. Nucleic Acids Res. 2016;44(D1):D279-85.

\section{Submit your next manuscript to BioMed Central and we will help you at every step:}

- We accept pre-submission inquiries

- Our selector tool helps you to find the most relevant journal

- We provide round the clock customer support

- Convenient online submission

- Thorough peer review

- Inclusion in PubMed and all major indexing services

- Maximum visibility for your research

Submit your manuscript at www.biomedcentral.com/submit
Biomed Central 